\title{
Juventud y Cooperativismo: Valoración del IMPacto de las Experiencias del Trabajo con Jóvenes en las Cooperativas de Colombia en la Sostenibilidad del Movimiento
}

\author{
Juan Carlos Mejía Cuartas \\ Soely Rúa Castañeda(*)
}

\begin{abstract}
Resumen: Las cooperativas son una manifestación empresarial de la economía solidaria y del cooperativismo como corrientes de pensamiento y acción. Ellas requieren desarrollar estrategias que garanticen la sostenibilidad, no solo de la empresa, sino de la identidad que guía sus acciones y desarrollo. Los avances en la ciencia administrativa han traído a las cooperativas prácticas que al ser implementadas han generado mejores resultados económicos y empresariales. Sin embargo, el movimiento cooperativo es cada vez más cuestionado en su compromiso con las prácticas éticas y de responsabilidad social. El presente artículo plantea que el carácter de cooperativista no se decreta, sino que debe formarse desde edades tempranas para así poder contar con asociados, dirigentes, empleados y ejecutivos convencidos de las ventajas de la forma cooperativa en la forma de generar el desarrollo empresarial y económico en sus comunidades de influencia. El presente artículo expondrá los resultados de un estudio desarrollado en el marco de la investigación "Modelo de inserción activa de jóvenes para la sostenibilidad del cooperativismo”, en el cual se consultaron 137 cooperativas de todo Colombia, de las cuales se seleccionaron 31 experiencias relevantes que posibilitaron el desarrollo de conclusiones en términos de razones, estrategias e impactos del trabajo con jóvenes en las cooperativas.
\end{abstract}

Palabras-clave: Juventud, Cooperativas, Economía Solidaria, Sostenibilidad.

\begin{abstract}
Cooperatives are Business expressions of the solidarity economy and cooperativism as streams of thoughts and action. They need to come up with strategies to guarantee their sustainability, not only of the business aspect, but also of the identity that leads their actions and development. The progress made by the management sciences has brought to cooperatives practices that have improved their business performance. However, the cooperative movement is being more and more questioned about its commitment to ethical practices and social responsibility. This article states that the cooperative characteristic is not spontaneous; therefore it needs to be seeded in early stages of life in order to count in the future with members, directors, employees and executives convinced of the advantages of the cooperative figure, in terms of generating economicentrepreneurial development in their communities. This article presents the results of the research "Model for cooperative sustainability through active youth inclusion", in which 137 cooperatives all over Colombia participated and out of this group 31 were selected for further studies related to their relevant experiences in youth work.
\end{abstract}

Keywords: Youth, Cooperatives, Solidarity Economy, Sustainability.

(*) Pesquisadores do Grupo GESTOR - Universidad de Antioquia (Medellín, Colômbia). Juan Carlos Mejía Cuartas, Email:jmejiacu@une.net.co. Soely Rúa Castañeda,E-mail: soerua@tutopia.com. Recebido em: 20.11.08 e aceito em: 3.12.08. 
"Lejos de las fiebres embrujadas de la infancia, de las esperanzas atormentadas de la adolescencia, y de las felicidades evanescentes de la juventud, pero teniendo todas esas edades presentes, la edad mayor puede aprender a convertir todo aquello que nos fue dado para que lo perdiéramos, en una posesión que no nos abandone, en un saber, en los secretos de una experiencia, en algo que puede compartirse con los otros. Por eso se equivocan los pueblos que creen que pueden renunciar a la vejez y a sus tesoros. Por eso los pueblos más sabios son aquellos en los cuales la pasión que va en busca de experiencia sabe dialogar con la experiencia que no ha perdido su pasión.”

William Ospina

\section{INTRODUCCIÓN}

Quizás una de las evidencias de evolución de la humanidad, es la proliferación de las reflexiones sobre el desarrollo y lo que él representa. En la actualidad se habla de las distintas dimensiones del desarrollo y de los alcances que debe tener en términos de mejoramiento de la calidad de vida de los seres humanos, que trasciende la concepción clásica que lo igualaba al crecimiento.

Son precisamente estas reflexiones las que han motivado la búsqueda de caminos alternativos que den respuesta a los retos que presentan las tradicionales dinámicas de concentración de la riqueza y las oportunidades. Hoy el mundo presenta un panorama nunca antes visto en donde hay más personas que nunca, más riqueza económica que nunca, más ricos que nunca, pero también más pobres que nunca; las brechas sociales son innegables e inocultables.

De acuerdo con los datos presentados por Naciones Unidas a lo largo de los años, es innegable que, a la luz de indicadores como mortalidad infantil y analfabetismo, el mundo ha venido avanzando en resolver problemáticas de orden social importantes como la salud y la educación. Sin embargo, quedan retos importantes en términos de distribución de la riqueza y de las oportunidades de acceso a otras dimensiones del desarrollo.

Expertos en diferentes disciplinas, de fuentes oficiales y no oficiales, han venido haciendo un seguimiento del fenómeno de las asimetrías en el desarrollo, el cual se da, no solo entre países sino al interior de cada país. Este seguimiento ha llevado a una mayor constatación y comprensión del problema del desarrollo y a un pronunciamiento recogido en la llamada Declaración del Milenio, carta emitida por la Asamblea de las Naciones Unidas, que expresa los objetivos de desarrollo que deben ser cumplidos por la humanidad antes del año 2015 y con los que los gobiernos de cada país se comprometen a alcanzar, bien por vía directa de la acción estatal o bien a través de estrategias como la cooperación internacional.

El caso es que las asimetrías en la distribución del ingreso son cada vez más evidentes, indicando que el desarrollo en todas sus dimensiones resulta un privilegio de minorías. Este escenario precisa que se fortalezcan estrategias de todo orden que impliquen un mayor acceso a las oportunidades y así democratizar el desarrollo.

El cooperativismo es un movimiento económico empresarial, democrático y socio cultural, que se materializa en las cooperativas y es una expresión de la llamada economía solidaria. Su propuesta organizativa e identitaria, alberga principios y valores que dan 
respuesta a las necesidades de desarrollo incluyente y dignificante para la humanidad. De igual forma, ha logrado importantes desarrollos empresariales que lo ponen hoy día a la par de los grandes poderes económicos mundiales y que han demostrado que puede generarse un desarrollo alternativo al que propone el modelo imperante.

Según datos de la Alianza Cooperativa Internacional, las cooperativas juntas a nivel mundial congregan a más de 800 millones de personas y emplean a un $20 \%$ más que todas las multinacionales juntas. La facturación en 2005 de las 300 cooperativas más grandes, le implicó ser la novena economía del mundo, comparable con Brasil o Canadá(1).

Son innegables los avances tanto empresariales como sociales de este modelo. Sin embargo, el cooperativismo enfrenta una gran amenaza que mina sus potencialidades de ser una respuesta integral al reto del desarrollo tal como se concibe hoy día. Su dirigencia y membresía está constituida por personas muy mayores, generalmente hombres que superan los 50 y hasta 60 años de edad, que en la mayoría de los casos no han hecho un proceso de transferencia de visión y concepción del movimiento a nuevas generaciones, quienes a su vez lo ven como algo anticuado y cerrado al que no tienen acceso y que no despierta mayor interés.

Algunos jóvenes que se han vinculado como empleados lo han hecho desde una dimensión técnica que soporta la parte empresarial, sin una comprensión de las dinámicas políticas, sociales y culturales que diferencian a las cooperativas de otras formas organizativas y económicas. Queda la pregunta entonces por la sostenibilidad del cooperativismo como propuesta que integra lo económico, la empresa, el ejercicio de la democracia a través de la participación en diferentes formas y niveles, la incorporación de aspectos culturales como la solidaridad, la equidad, el respeto, la diversidad, la educación y otros aspectos de orden social que llevan a las personas que interactúan dentro de la cooperativa a un estadio superior de la dignidad humana.

Es por ello que se propuso la realización de una investigación rigurosa que estudie las dinámicas juveniles y los intereses de los jóvenes al acercarse a una cooperativa, que a su vez se contrasten con las opciones reales que están dando estas empresas para que ellos sean parte activa en las decisiones y las proyecciones del movimiento.

El presente artículo expondrá brevemente los lineamientos teóricos que sustentaron la construcción de la investigación, seguidamente expondrá la metodología que se siguió y por último, expondrá los hallazgos encontrados en la variable de impacto, una de de las variables identificadas dentro del estudio.

\section{La Sostenibilidad del Cooperativismo}

La investigación basó su construcción en tres ejes conceptuales: La economía solidaria, la sostenibilidad y juventud, bajo la premisa de que las cooperativas se hacen sostenible en el tiempo a través del trabajo propenda por la inserción de jóvenes. Los planteamientos

(1) ACI. Global 300 Report. Ginebra. 2007. 
ampliamente desarrollados en el informe final de la investigación se resumen en el siguiente Figura:

\section{Figura 1 - Modelo de sostenibilidad del cooperativismo}

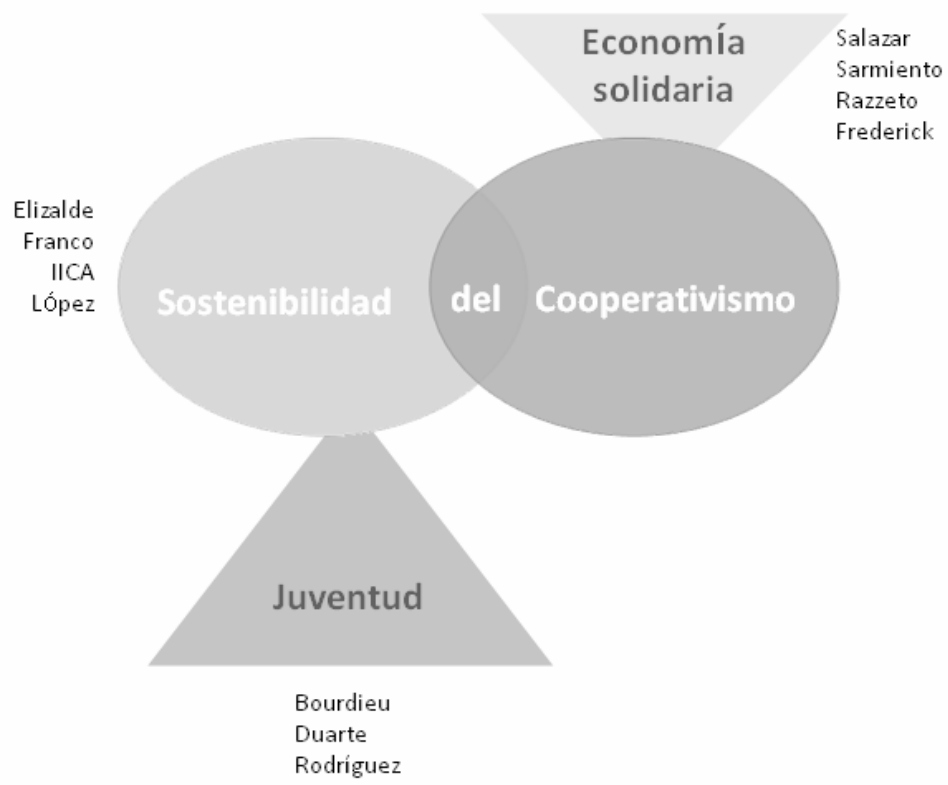

Figura 1 ilustra la sostenibilidad del cooperativismo como el centro del problema, siendo el cooperativismo una expresión de la economía solidaria y la juventud un soporte para la sostenibilidad.

Para entender la identidad cooperativa, y en general el cooperativismo, es preciso conocer y comprender el concepto de economía solidaria, pues este es la lógica a partir de la cual se construye el cooperativismo como corriente de pensamiento y acción. Una vez lograda esta comprensión se desarrollará la identidad cooperativa como la materialización de los preceptos de la economía solidaria

Razeto (1996) plantea que la economía de solidaridad no es la negación de la economía de mercado, pero tampoco es su simple reafirmación. Si bien la generación de la riqueza requiere de la optimización de los recursos para lograr la efectividad en la generación de riqueza que produzca bienestar, existen paradigmas que van más allá del simple hecho del crecimiento y que incorporan visiones más sistémicas; es decir, que reúnen las múltiples dimensiones del desarrollo sostenible ${ }^{(2)}$.

La fraternidad y la solidaridad son garantes para que el proceso económico se geste de manera eficiente para toda la sociedad. Además de la riqueza material que se deriva del trabajo colectivo, su aporte a la sociedad incorpora elementos de cohesión social que hacen posible que el desarrollo se de de una manera armónica y sostenible, gracias a que, a través

(2) Concepto analizado en el capitulo dedicado a la sostenibilidad. 
del trabajo, la comunidad genera relaciones que conforman un tejido social sobre el que se soportan las construcciones económicas, políticas y culturales.

Libardo Sarmiento Anzola ${ }^{(3)}$ en ponencia realizada en la cuarta versión del Foro de Solidaridad ${ }^{(4)}$ presentó la economía solidaria como aquella economía que busca la dignidad humana a partir de la integración de tres ejes fundamentales: La solidaridad; entendida como la adhesión a una causa, la asociatividad, entendida como la congregación de personas para alcanzar un fin común y finalmente la autogestión; entendida como la participación en el propio desarrollo personal y comunitario.

Se requiere de una coordinada y complementaria gestión de estos tres factores, pues no es posible construir economía solidaria a partir de un solo de ellos, pues se generan distorsiones que son lesivas tanto para el individuo como para la comunidad y el tejido social sobre el que reposa.

Cuando la solidaridad se encuentra con la autogestión, se generan dinámicas de mercado en función de la sociedad y que pueden derivarse incluso en construcciones de estado o gobiernos que efectivamente respondan a intereses y dinámicas colectivas. Cuando la solidaridad se encuentra con la autogestión se posibilita la construcción de organizaciones democráticas, plurales e incluyentes, construidas con la participación de todo el colectivo. Finalmente cuando la asociatividad se encuentra con la autogestión, el resultado es un trabajo que genera riqueza y apropiación social.

Es preciso entender esta visión de desarrollo colectivo que La Economía Solidaria, como corriente de pensamiento y acción, propone. Tal como lo plantea Dubois (2005) "Si no se hace este ejercicio, se corre el riesgo de promover acciones dependientes de otras concepciones del desarrollo y que favorecen a los intereses de determinados sectores que no nos garantizan ser los prioritarios".

Ahora bien, la pregunta es ¿cómo hacer de la propuesta cooperativa, una alternativa sostenible en el tiempo?

En virtud de responder a este cuestionamiento, se abordó concepto de sostenibilidad, para lo cual fueron fundamentales los aportes de Franco (2005), Elizalde (2003) e IICA (2007).

Podría definirse la sostenibilidad como la permanencia y desarrollo de LA VIDA en el tiempo y el espacio; y de los entornos que la gestan, la soportan y la continúan.

En primera instancia esta definición se identifica con el concepto de Brand al poner a LA VIDA, en su sentido más amplio. De esta manera se hace referencia a los seres vivos individuales, a los seres colectivos, a los espacios, a las ideologías y a los sistemas que se componen con individuos y colectivos, tales como organizaciones, países, familias, etc., y los entornos que la circundan, la propician y la soportan.

Como segundo elemento de la definición permanencia y el desarrollo en el tiempo y el espacio, son indicadores de la durabilidad y validez, entendiendo el concepto de

(3) Filósofo, Economista y Master en Teoría Económica de la Universidad Nacional de Colombia.

(4) Evento anual realizado en la ciudad de Medellín, Colombia y organizado por confiar - Cooperativa Financiera, que promueve la cultura de la economía solidaria. 
desarrollo como evolución; es decir, como mejoramiento de una condición inicial a una condición superior que supone bienestar. La permanencia y el desarrollo de la vida, implican la permanencia y desarrollo de los entornos, de la alteridad circundante, de los contextos en donde dicha vida se origina. Así mismo, de aquellos entornos que hacen posible que una vez originada esa vida permanezca y continúe.

La sostenibilidad tiene diferentes dimensiones, plasmadas en el siguiente Cuadro.

Cuadro 1 - Dimensiones de la sostenibilidad

\begin{tabular}{|l|l|l|}
\hline \multicolumn{3}{|c|}{ SOSTENIBILIDAD } \\
\hline DIMENSIONES & \multicolumn{2}{|c|}{ RELACIONES QUE LA CONSTITUYEN } \\
\hline $\begin{array}{l}\text { Socio- } \\
\text { ambiental }\end{array}$ & $\begin{array}{l}\text { Naturaleza y el ambiente construido } \\
\text { yumana. } \\
\text { humodificado por la intervención }\end{array}$ & $\begin{array}{l}\text { Uso racional de los recursos naturales. } \\
\text { Buscar una mejor distribución territorial de } \\
\text { los asentamientos humanos y de las activi- } \\
\text { dades económicas }\end{array}$ \\
\hline Cultural & $\begin{array}{l}\text { Los sistemas de lenguaje, las creen- } \\
\text { cias, los valores y sus consecuentes } \\
\text { comportamientos. }\end{array}$ & $\begin{array}{l}\text { Buscar las raíces endógenas de los procesos } \\
\text { de modernización }\end{array}$ \\
\hline Política & $\begin{array}{l}\text { El Estado, las relaciones de poder, la } \\
\text { legitimidad y la gobernabilidad. }\end{array}$ & $\begin{array}{l}\text { Propiciar la participación de los grupos y de } \\
\text { las comunidades locales en las definiciones } \\
\text { de prioridades y metas a ser alcanzadas. }\end{array}$ \\
\hline Económica & $\begin{array}{l}\text { El mercado, el crecimiento, la produc- } \\
\text { ción de bienes y servicios, el consumo } \\
\text { y el ahorro, y la inversión. }\end{array}$ & $\begin{array}{l}\text { Asignar y gestionar eficientemente los Re- } \\
\text { cursos. }\end{array}$ \\
\hline Social & $\begin{array}{l}\text { La sociedad civil y los actores y mo- } \\
\text { vimientos sociales }\end{array}$ & $\begin{array}{l}\text { Garantizar equidad en la distribución de } \\
\text { ingresos y de bienes. }\end{array}$ \\
\hline
\end{tabular}

FUENTE: Elaboración propia a partir de los planteamientos de Elizalde (2003), Franco (2005), e IICA (2007).

La sostenibilidad se da en la medida en que las generaciones presentes y futuras participan de las nuevas construcciones. La educación, la formación y la información son claves para la participación efectiva y estas deben darse de manera permanente en formatos digeribles y dosis adecuadas para todos los participantes; en función de recoger de ellos todas contribuciones que sus condiciones les permitan y de potencializar otras contribuciones que se puedan desarrollar a través del ejercicio mágico del aprendizaje que se deriva de la experiencia colectiva.

López (2003) agrega que "esta educación deberá manifestar un interés especial por la creación, innovación, experimentación, observación, análisis crítico, riesgo, cambio, opciones nuevas y siempre dentro de una gran flexibilidad que abra espacios reales y concretos al ‘aprendizaje colectivo', evitando la imposición y la sola transmisión”. La educación para la sostenibilidad debe ser formadora de nuevas interpretaciones de la realidad planetaria e inspiradora de nuevas creaciones, a partir las realidades existentes y las por construir. Esta es posible con la participación de los líderes actuales quienes, a través de un diálogo intergeneracional se transmitan los conocimientos y aprendizajes expresos y tácitos adquiridos, a los nuevos líderes de punta y de base en cuyas manos quedan las nuevas construcciones.

El reto de la sostenibilidad es fundamentalmente un reto de aprendizaje, un proceso que requiere tanto "cambios externos" como métricas nuevas, "cambios internos" en lo que respecta a los supuestos que se dan por sentados y los modos de operar. (SENGE, 2007) 
En resumen, hacer de la Economía Solidaria una propuesta sostenible requiere de la formación de una conciencia, si bien de las generaciones presentes, más aún de las futuras generaciones. Esta formación debe abarcar todas las dimensiones de sostenibilidad anteriormente planteadas y en todos los aspectos característicos de las cooperativas como expresión de la economía solidaria.

\section{Metodología}

En el contexto de la representación de los Jóvenes en la mesa directiva mundial de la Alianza Cooperativa Internacional ${ }^{(5)}$, se quiso indagar sobre el estado de las experiencias de cooperativismo y juventud, en miras a desarrollar un modelo que orientase a las cooperativas a desarrollar iniciativas en materia de cooperativismo y juventud. Para ello se planteó un proyecto de investigación que inicialmente abarcó a las cooperativas Colombianas y que se proyecta replicarse en otros países en donde haya cooperativas.

La investigación tuvo un alcance exploratorio-descriptivo y tomó como objeto de estudio las cooperativas colombianas confederadas en Confecoop; es decir 710 cooperativas del universo de 6810 cooperativas presentes en el país.

Una vez elaborado el Marco Teórico, se desarrolló un trabajo de campo en dos fases. La primera fase consistió en la aplicación de un instrumento en formato virtual, dirigido a todas las cooperativas confederadas, de las cuales respondieron 137. El objetivo era identificar cuáles de las cooperativas tenían trabajo con jóvenes y algunas características a profundidad que permitieran seleccionar a un grupo de cooperativa a estudiar a profundidad en una segunda fase.

Se entrevistaron un total de 22 de las 31 cooperativas, se descartaron 8 por la dificultad de coordinar los horarios para la entrevista o bien no respondían a las citaciones y llamadas. Una se auto-descartó por no considerarse una experiencia relevante en materia de cooperativismo y juventud.

Las experiencias estuvieron ubicadas en 15 ciudades y municipios de 10 departamentos de Colombia. Se realizaron un total de 75 entrevistas a 191 personas, dentro de las que se encontraban gerentes (15), directivos (14), responsables (33), colaboradores (7) y jóvenes (119). Las entrevistas se realizaron entre el 16 de noviembre y el 16 de diciembre de 2007 y entre el 14 de enero y el 26 de febrero de 2008.

En la segunda fase se identificaron 5 variables que fueron:

Motivación: explica las razones, tanto institucionales como personales, que tienen los actores involucrados en procesos cooperativos con jóvenes para hacer su participación.

En el campo institucional se exponen las situaciones y razones por las cuales las cooperativas decidieron iniciar las actividades y procesos con jóvenes. Igualmente menciona el proceso de la organización para tomar las decisiones concernientes al tema de juventud.

(5) Cargo ocupado por Juan Carlos Mejía de 2005 a 2007 
En cuanto lo personal describe las razones o los medios que acercan a los jóvenes a la cooperativa y las razones por las cuales los jóvenes permanecen.

Oferta: Esta variable se compone de dos elementos. Un primer elemento es la oferta que los jóvenes que participan en actividades alrededor de la cooperativa, encuentran para ellos en el contexto que habitan o con el que interactúan, adicionales a las que ofrece la cooperativa. Este aspecto fue denominado Oferta del Entorno.

Un segundo elemento constitutivo de la oferta es el que hace referencia las actividades y programas que las cooperativas tienen para jóvenes, el cual fue llamado Oferta Cooperativa.

Evidencias de la política: Esta variable expone los indicadores de la existencia de una política que oriente el trabajo con jóvenes en las cooperativas.

En los testimonios recogidos en el estudio se pudo identificar que hay una gran presencia de criterios de orden administrativo, tales como edades máximas y mínimas, tipo y cantidad de personas a invitar, mas no necesariamente de políticas de gran alcance que den cuenta de una claridad en este aspecto.

Para facilitar el análisis, se definieron 3 indicadores de evidencia de la política, determinados a juicio de los investigadores, basados en su experiencia y conocimiento. Esto son: Presupuesto, documentos que contengan la política y Estructura.

Valoración del beneficio: Esta variable describe las percepciones que directivos, gerentes y responsables tienen de los beneficios del trabajo con jóvenes.

Los beneficios percibidos se ubican en las áreas de beneficios para el individuo, para la familia, para la institución, para el sector, para la sociedad y un beneficio comercial.

Impacto: En vista de que se pudo identificar que las cooperativas adolecen de mecanismos para hacer una medición y seguimiento del impacto de las estrategias de trabajo con jóvenes, con base en la fundamentación teórica que soportó el estudio, se establecieron tres variables para ser observadas en los jóvenes y así identificar cuál es el avance como institución y como sector en materia de sostenibilidad que fueron la participación e incidencia en la cooperativa, la incorporación de la conciencia cooperativa y finalmente la proyección del joven en la cooperativa.

Los hallazgos en esta última variable son objeto del presente artículo, pues dan cuenta de la efectividad de las estrategias en materia de sostenibilidad y la coherencia entre acciones y discursos de dirigentes y administrativos de las cooperativas.

\section{IMPACTO}

Una medición y seguimiento del impacto de las estrategias de trabajo con jóvenes. En vista de esto, con base en la fundamentación teórica que soportó el estudio, se establecen tres indicadores para ser observados en las experiencias, permitiendo así identificar cuál es el avance como institución y como sector en materia de sostenibilidad. Estos son la participación e incidencia en la cooperativa, la comprensión, práctica e identidad solidariacooperativista y finalmente la proyección del joven en la cooperativa. 


\section{Participación e incidencia}

Antes de entrar en materia, es importante hacer la precisión sobre los conceptos de participación e incidencia. La participación hace referencia a la manera cómo el joven se involucra en la dinámica de la cooperativa. Esta tiene distintos niveles que indican qué tan identificado se siente con el espacio de la cooperativa, o bien qué tanto permite la cooperativa la participación.

En la búsqueda por entender las dinámicas de participación de los asociados, CONFIAR ha definido los tipos de participación que ellos hacen y que se presentan para efectos del análisis de este aspecto. Ellos son: Asociado Usuario, Asociado Promotor, Asociado Delegado y Asociado Dirigente.

El primero se caracteriza por una relación de cliente, donde su participación se limita al uso o consumo de los bienes o servicios de la cooperativa. En segundo lugar estará el asociado promotor, quien además de utilizar los servicios de la cooperativa, alienta a otras personas a vincularse a ella. También está el asociado delegado, quien además de participar en las dos formas anteriores conoce más a profundidad la cooperativa, hace seguimiento de su comportamiento y busca intervenir en la toma de decisiones sobre las políticas de desarrollo institucional. Por último, se encuentra el asociado dirigente, quien está a la cabeza de la estructura social y toman las grandes decisiones institucionales.

La incidencia, hace referencia a la afectación que tiene la participación del joven en el devenir de la cooperativa; es decir, qué tanto se tiene en cuenta su punto de vista.

Con esta introducción, se puede afirmar que en la gran mayoría de los casos, los jóvenes participan desde los niveles de usuario y promotor; dado que se limitan a ser beneficiarios de los programas diseñados para ellos e interlocutan con la cooperativa en función de esas actividades o procesos. En algunos casos, los jóvenes cumplen un papel más activo en la promoción de la cooperativa, en tanto intervienen como apoyo logístico de algunas actividades que esta adelanta. En cooperativas como CONFIAR, BELÉN, COOFISAM y COONALTRAGAS los jóvenes alcanzan incluso niveles de delegados. Solo en COOLÍDERES y CRESER los jóvenes logran actuar como dirigentes.

Ya en términos de incidencia, es claro que solo en dos de las cooperativas estudiadas (COOLÍDERES y CRESER), la participación del joven es determinante en la vida y proyección organizacional y coincide con que son las dos cooperativas de jóvenes. En aquellas cooperativas que han abierto el espacio para que los jóvenes participen además como delegados, se percibe una intencionalidad de formar al joven como actor político dentro de la cooperativa y así como una relación joven-adulto positiva. En las demás experiencias, la generalidad es un distanciamiento del mundo joven del adulto.

Existen cooperativas en las que los jóvenes juegan un papel determinante en la planeación, selección y seguimiento de actividades constitutivas de su proceso de formación cooperativa, como es el caso de COOTRAIM, COOFISAM, FUNDAUTRAHUILCA, CONFIAR, CRESER, COOLÍDERES y LA EQUIDAD, en cuanto a lo que la Red Juvenil Cooperativa se refiere. Los asuntos económicos no son territorio de deliberación de los jóvenes. Tan sólo Coofisam, Creser y Coolíderes han creado estos espacios de discusión. Este tema es restringido para la dirección o administración de la cooperativa. 
Otros grupos de jóvenes argumentan que su participación se limita a la evaluación de las actividades ejecutadas. Este comportamiento es característico de las cooperativas donde los programas para jóvenes son actividades más que procesos formativos constantes. En la mayoría de los casos se les consulta su opinión sobre las actividades a desarrollar, pero como dice un testimonio "al final siempre mandan los directivos".

Lo anterior no es necesariamente percibido por los jóvenes como algo negativo, pues se sienten tenidos en cuenta y al parecer no tienen expectativas de mayor participación, dado que en la gran mayoría de los casos, se está ante la presencia de niños y adolescentes.

Es precisamente esta característica del trabajo con jóvenes la que explica los niveles de participación y, por ende, de incidencia en la cooperativa. Al estar la oferta limitada a los adolescentes, los jóvenes de la cooperativa no cuentan con las condiciones personales ni académicas para hacer una participación en los niveles de incidencia. Queda la pregunta por los espacios de participación para los jóvenes más adultos, quienes tendrían la posibilidad de hacer una participación distinta y que es, en últimas, la que contribuye a la sostenibilidad.

\section{COMPRENSIÓN, PRÁCTICA E IDENTIDAD SOLIDARIA COOPERATIVISTA}

Un primer aspecto de este indicador tiene que ver con el conocimiento, la comprensión y la percepción de la diferencia entre la cooperativa y las organizaciones no cooperativas.

Para este análisis, se tomará como referencia el planteamiento hecho por Frederick ${ }^{(6)}$ (1997), quien resume en tres principios básicos la diferencia entre la cooperativa de otro tipo de negocios, que son el usuario beneficiario, el usuario dueño y el usuario que controla.

\section{EL PRINCIPIO DEL USUARIO BENEFICIARIO}

Los miembros se unen en una cooperativa para obtener servicios que de otra manera no estarían disponibles: para obtener servicios públicos en el momento necesario, acceso al mercado y por otras razones de mutuo beneficio. Actuar conjuntamente les da a los miembros la ventaja de las economías de escala y de poder de negociación. Ellos se benefician de tener estos servicios disponibles, en proporción al uso que hagan de ellos.

Los miembros también se benefician al compartir las ganancias del negocio que se conduce en forma cooperativa. Cuando las cooperativas generan márgenes por operaciones eficientes y agregación de valor a productos, estas utilidades son devueltas a los miembros en proporción al uso de la cooperativa. Sin la cooperativa estos fondos irían a otros intermediarios.

Este principio es uno de los más recurrentes en las experiencias visitadas. Los jóvenes claramente evidencian la parte social como un aspecto positivamente diferenciador, que a su vez se convierte en un factor de motivación.

(6) FREDERICK, Donald A. Co-ops 101: an introduction to cooperatives, p. 5, 6. 1997. 


\section{EL PRINCIPIO DEL USUARIO PROPIETARIO}

La cooperativa pertenece a quién la usa. Al ser dueños de los activos, los miembros tienen la obligación de proveer la financiación de acuerdo con el uso para mantener a la cooperativa funcionando y creciendo.

Este principio no es tan visible en el discurso de los jóvenes como el anterior. Sin embargo, a partir de sus testimonios, se evidencia que está tan incorporado que no hay necesidad de mencionarlo. Ellos saben que la cooperativa los beneficia porque sus padres o ellos mismos, son los dueños y se sienten como tal.

\section{EL PRINCIPIO DEL USUARIO QUE CONTROLA}

Como propietarios, un miembro de una cooperativa controla sus actividades. Este control se ejercita a través del voto en reuniones anuales e indirectamente por aquellos miembros elegidos a la junta directiva. Los miembros, en la mayoría de los casos, tienen un voto independientemente de la cantidad de capital del que son dueños.

El principio del control de los miembros es claro para los jóvenes, sobre todo por la directa relación que hacen con el ser dueño. Al igual que el principio de la propiedad, este está incorporado de una manera menos visible en el testimonio de los jóvenes. De todas maneras, se encontraron afirmaciones que dan cuenta de la claridad del contexto cooperativo en cuanto al control.

Otro aspecto, no tenido en cuenta por la definición de Frederick es lo referente a la práctica e identidad solidaria cooperativista, que tiene gran fuerza en los jóvenes participantes de las actividades y procesos de formación.

Los jóvenes se identifican con el cooperativismo, sus valores y principios y le dan una relevancia al asunto. Las experiencias alrededor de formación cooperativa en la academia tienen un matiz distinto al de las cooperativas con otras actividades económicas.

El Colegio Cooperativo Los Álamos en Bogotá, ha desarrollado experiencias en donde a través del proceso de una feria de emprendimiento con niños y jóvenes, incorporan actitudes y valores cooperativistas, aún si estos no son concientes. En esta experiencia se notó que los jóvenes no tienen una apropiación del discurso cooperativo (principios, valores, símbolos), pero cuando se les pregunta por su experiencia, ellos inmediatamente describen su cotidianidad eminentemente cooperativa, basada en proyectos y trabajo conjunto.

"No unimos para movernos fácilmente y para poner todos los ingredientes. Dar mucha plata, porque a veces uno solo de pronto no tiene la plata."

"Nos reunimos en una casa y ahí fue donde empezamos a ayudarnos y entre los tres decidimos qué íbamos a llevar.” 
"A nosotros siempre nos han dicho que trabajemos juntos y las actitudes giran conforme a eso."

"Al final de todo reuníamos toda la plata y la juntamos y la repartimos."

En otra experiencia, se encontraron testimonios alrededor de la cátedra cooperativa que ilustran el impacto de esta estrategia como formadora de consciencia cooperativa:

“yo le he oído ha los alumnos mamei... ellos dirán que esa clase para qué les sirve, no es como la matemática, la física, la química.”

"A veces no les llega absolutamente nada, ellos decían: la clase de cooperativismo que fastidio, que mamera."

"Los muchachos que llegan por primera vez a sexto a séptimo no les importa, pero los que vienen desde primaria a los niños se les da una formación diferente, entonces a ellos si les importa. Por ejemplo los colores, nosotros decimos: vamos ha hacer tal cosa con amarillo. $\mathrm{Ah}$ ! Si, amarillo porque representa el sol que alumbra, eso nos lo enseñaron en cooperativismo."

"Es diferente cuando están grandes. Cuando llegan a la fundación y vienen con una formación desde primaria, tienen otra perspectiva y eso es lo que quiere hacer la fundación creando la primaria. En noveno o en once eso no nos importaba, por ejemplo yo venia de otro colegio donde lo más importante era biología o matemáticas, en cambio aquí lo mas importante es proyecto social, cooperativismo y todo lo que tiene que ver con la parte social.”

COOEDUCAR

La formación en cooperativismo en niveles superiores también genera respuestas importantes en materia de consciencia cooperativa. Esta cooperativa, que dentro de sus líneas de negocio tiene una universidad, tienen un plan de formación en cooperativismo:

En el primer semestre vemos filosofía solidaria, ahí nos hablan de cooperativismo, que el cooperativismo: cuáles son los valores y los principios... En cuarto semestre ve uno administración de empresas de economía solidaria y ya ahí uno le va cogiendo la parte más legal, cómo se constituye una empresa cooperativa, las clases de cooperativa, las funciones, las personas que las integran...

Este programa les brinda mayor claridad frente a los mitos y realidades de la forma cooperativa, pues dicen: "Uno se imagina que todas las cooperativas son iguales: la cooperativa de transporte es como un fondo de empleados, hasta que uno ve esa cátedra y recibe esa información. Uno ya tiene sus diferencias que las cooperativas son diferentes, que los fondos mutuales y se va dando cuenta que hay muchas más de las que uno cree." 
Lo anterior lleva a pensar que la academia sí cumple un papel determinante en la formación de comprensión práctica e identidad solidaria cooperativista, pero las estrategias de formación deben ser acordes con la etapa que vive el joven, para que le encuentre pertinencia con su realidad y así ser efectivos en el propósito de cooperativizarlos.

\section{Proyección y oportunidades de MEJORAmiento}

Finalmente, se describen las propuestas de mejoramiento que los jóvenes hacen a la cooperativa, de acuerdo con las expectativas que tienen frente a su futuro dentro de ella.

Esta variable tiene dos componentes, uno es la proyección y el otro las expectativas que los jóvenes tienen de la cooperativa, tanto en el presente como en el futuro.

En el primer aspecto se identifican clara y explícitamente las intenciones de mantener la relación con la cooperativa y/o el sector; muchos de ellos con perspectivas de vinculación laboral. En este punto es importante resaltar que este deseo surge del compromiso con el cooperativismo más que de la necesidad de trabajo, porque allí encontraron un proyecto de vida, con unos adultos que son modelos de referencia positivos.

También los jóvenes se ven como multiplicadores de los procesos de los que se han visto beneficiados, con el fin de garantizar la sostenibilidad del programa del que han hecho parte.

En el segundo aspecto de esta variable, las oportunidades de mejoramiento, se identifican tres tendencias principales: Mayor participación de jóvenes en la toma de decisiones, mayor articulación con otros espacios dentro y fuera de la cooperativa y las propuestas concretas de actividades.

Se puede identificar que los jóvenes tienen aspiraciones de una mayor incidencia en la determinación de actividades e iniciativas, tanto de sus procesos de formación como cooperativista como de la cooperativa en general.

Otra expectativa que tienen los jóvenes es una mayor articulación y conocimiento de otros procesos con su misma cooperativa y con otras cooperativas, así como la participación e interacción con los adultos.

Por último, los jóvenes proponen crear proyectos en los cuales se sientan más cómodos, quieren emprender actividades de mayor responsabilidad para aumentar el nivel de compromiso hacia la cooperativa y que ésta a su vez esté presente en dichas actividades en el papel de respaldo.

Estas propuestas surgen debido a que los jóvenes que comienzan a alejarse de la cooperativa, principalmente por el comienzo de una vida universitaria, sienten que el trabajo que han venido haciendo no se puede echar a perder.

\section{Proceso}

Un aspecto importante a tratar dentro de esta variable, es el proceso que se lleva en la cooperativa para la toma de decisiones con respecto al trabajo con jóvenes. Al respecto se identificaron dos tendencias: de arriba hacia abajo y de abajo hacia arriba. 
La tendencia más predominante es de arriba hacia abajo, que indica que las ideas surgen en la dirección o administración de la cooperativa. Normalmente el consejo encarga al comité de educación y este a su vez se apoya en la persona del área social o en la fundación, en caso de haberla, para la ejecución de la iniciativa. En este tipo de situaciones, los jóvenes asumen un papel de beneficiarios y como tal su intervención en el proceso de decisiones es limitada. Cabe decir que esta situación coincide con la percepción que los mismos jóvenes tienen sobre su incidencia en las decisiones de la cooperativa, e incluso de su mismo proceso.

La otra tendencia identificada es de abajo hacia arriba. Ello implica que las propuestas vienen directamente del grupo beneficiario y que llegan a niveles superiores, básicamente para apoyo y/o aprobación presupuestal. Si bien esta situación no es la más recurrente, es interesante ver cómo los procesos de empoderamiento de los jóvenes, efectivamente hacen que ellos propongan iniciativas, no solo para su propio proceso, sino para otras actividades de la cooperativa, en las cuales ellos se vinculan de manera activa, tales como son los días de la familia, actividades de beneficencia y navideñas, entre otras.

En síntesis, sI bien no son abundantes las experiencias de trabajo con los jóvenes en las cooperativas de CONFECOOP en Colombia, sí existen experiencias diversas, con presencia en casi todas las regiones de la geografía nacional, que sirven de referencia para el desarrollo de actividades y posibilitan la reflexión sobre la estrategia de trabajo con jóvenes en las cooperativas para el aseguramiento de su sostenibilidad.

\section{CONCLUSIONES SOBRE EL IMPACTO}

El impacto debe medirse y dada la ausencia de indicadores que de cuenta de esta medición se proponen la participación e incidencia, la conciencia cooperativa y la proyección a futuro del joven dentro del cooperativismo.

\section{PARTICIPACIÓN E INCIDENCIA}

En la mayoría de los casos los jóvenes participan, en el sentido de que hay una dinámica en la cual ellos son consultados. Sin embargo, queda una pregunta frente al tema de la incidencia, pues sus aportes poco contribuyen a las dinámicas de la cooperativa. Este puede suceder por varias razones:

- En principio las actividades son desarrolladas en su mayoría con adolescentes o niños, quienes no cuentan con la suficiente preparación ni experiencia para hacer contribuciones que puedan incidir en la organización.

- Otro factor de capital importancia, es que los jóvenes no son vistos como pares más bien, son vistos como una población vulnerable y por tanto más pasiva en cuanto a la recepción de beneficios por parte de una población en mejores condiciones, concretamente, los adultos. Esto imposibilita una interlocución válida con el mundo adulto, desde un lugar de igualdad en dignidad y capacidad, lo que no significa desconocer 
las diferencias generacionales, pero lo que se busca es que esta condición no se vuelva factor determinante para la subvaloración de los aportes de los jóvenes.

\section{LA CONCIENCIA COOPERATIVA}

La academia cumple un papel determinante en la formación de consciencia cooperativa, pero las estrategias de formación deben ser acordes con la etapa que vive el joven, para que le encuentre pertinencia con su realidad y así ser efectivos en el propósito de formarles como cooperativistas.

A partir de estos análisis se pudiera proponer un criterio metodológico que sería:

\section{- CON NIÑOS, JUGAR AL COOPERATIVISMO}

En dos de las experiencias hubo testimonios que indicaban que, particularmente con niños y niñas, el tema era atractivo cuando se abordaba desde la lúdica.

\section{- CON ADOLESCENTES, EJERCITARSE CON EL COOPERATIVISMO}

La vivencia de lo cooperativo; es decir, donde se palpe lo económico, lo democrático y lo social-cultural de manera simultánea, adquiere una mayor relevancia en edades más adolescentes. Esto porque los jóvenes son más demandantes de acciones que de discursos. Acciones que le den validez a sus búsquedas, pero que los reivindiquen como seres responsables, inteligentes y propositivos. En esta etapa, los jóvenes están más dispuestos a participar de propuestas acompañadas por la cooperativa que materialicen la ideología cooperativista.

- CON JÓVENES ADULTOS, EMPRENDIMIENTOS COOPERATIVOS CONCRETOS DE PROTECCIÓN ECONÓMICA Y PROFESIONAL

En la etapa de educación superior el rol de los jóvenes cambia en el desarrollo de las iniciativas de formación cooperativista, ya que con el tiempo han ganado habilidades, destrezas y conocimientos. Esto es una situación que las propuestas de procesos juveniles en esta etapa deben tener en cuenta y no se deberían circunscribir solo a los temas de emprendimiento. Si bien el empleo es muy importante las necesidades en esta etapa de la vida van más allá de lo meramente económico.

\section{LA PROTECCIÓN A FUTURO}

Los jóvenes que se han involucrado activamente con las iniciativas de proceso tienen aspiraciones de una mayor incidencia en la determinación de actividades e iniciativas, tanto de sus procesos de formación como cooperativista como de la cooperativa en general. De igual forma aspiran a una mayor articulación y conocimiento de otros procesos dentro de su misma cooperativa, como con otras cooperativas, así como proponen crear proyectos en los cuales se sientan más cómodos, quieren emprender actividades de mayor responsa- 
bilidad para aumentar el nivel de compromiso hacia la cooperativa y que ésta a su vez esté presente en dichas actividades en el papel de respaldo.

\section{Conclusiones}

El trabajo con jóvenes en las cooperativas es un asunto de importancia estratégica, dado que el cooperativismo es una ideología y, como tal, habita en las personas. Si no hay una permanente seducción de nuevos convencidos, éste necesariamente tiende a desaparecer, aún si las empresas cooperativas persisten.

Las acciones en materia de trabajo con jóvenes en las cooperativas están centradas en niñez y adolescencia. Preocupa la ausencia de estrategias de vinculación de jóvenes adultos a los procesos participativos de las cooperativas, pues finalmente ellos son quienes tienen la mayor posibilidad de aporte al desarrollo de las cooperativas.

A pesar de que no hay una intención generalizada de profundizar en estrategias que busquen la inserción activa de jóvenes en las cooperativas o en el cooperativismo, el discurso cooperativo tiene un poder de atraer a los jóvenes y este es un punto a favor. Los jóvenes que se acercan a las cooperativas son receptivos de la propuesta identitaria y buscan contribuir en su construcción y fortalecimiento.

Si bien los discursos de gerentes y directivos cooperativos dan cuenta de una comprensión de la importancia y trascendencia de trabajar el tema de juventud de manera seria e intencionada, salvo excepciones, la realidad muestra que las cooperativas están centradas en la subsistencia organizacional y el desarrollo empresarial, y lo que se evidencia es que a la juventud no se le ve como un asunto estratégico en términos de sostenibilidad.

Se encontró que las iniciativas en materia de juventud son más intenciones personales, o a lo sumo institucionales. Como sector, en Colombia el tema es prácticamente invisible.

En términos generales, la sostenibilidad del cooperativismo está amenazada por el descuido de las dimensiones culturales y políticas. Culturales porque las nuevas generaciones están siendo vinculadas como clientes, en el caso de los asociados, o técnicos, en el caso de los empleados. Ellos desconocen el transfondo identitario que subyace en la propuesta cooperativa. En lo político, las dinámicas participativas están siendo ejecutadas por las mismas personas durante años, cerrando la posibilidad de inclusión de nuevas visiones y personas. En suma, podrá permanecer la cooperativa como empresa, pero hay un alto riesgo de que quienes la asuman a futuro desprecien el espíritu solidario que la originó.

\section{REFERENCIAS BIBLIOGRÁFICAS}

ACI. Global 300 Report. Ginebra, 2007.

AGUILERA, Oscar. Tan jóvenes, tan viejos: los movimientos juveniles en el Chile de hoy. Santiago de Chile: Instituto Nacional de la Juventud, 2003.

ARANGO, Miguel et al. Reflexiones sobre el aporte social y económico del sector cooperativo colombiano. Bogotá: Fedesarrollo — La Equidad Seguros — Fundequiad, 2005. 
AXELROD, Robert. The evolution of cooperation. Capítulos 1, 7 y 8. Seatle: Basic Books, 1984.

BALARDINI, Sergio. Políticas de juventud: conceptos y la experiencia argentina. Políticas públicas de juventud en América Latina. Viña del mar: CIDPA, 2003.

BONILLA, Wilfer. La condición social de juventud. En: Memorias inéditas del Seminario de Investigación sobre Producciones y prácticas culturales juveniles en Medellín. Medellín, Fundación Universitaria Luis Amigó e Instituto de Juventud XXI, 1998.

BOURDIEAU, Pierre. La juventud no es más que una palabra. Sociología ejecutiva. México: Grijalbo, 1990.

CAREW, A. L.; MITCHELL, C. Teaching sustainability as a contested concept: capitalizing on variation in engineering educators' conceptions of environmental, social and economic sustainability. Journal of Cleaner Production, n. 16, 2008. Disponible en: < http://www.sciencedirect.com>.

CARVAJAL, Álvaro. Teorías y modelos: formas de representación de la realidad. Revista Comunicación. San José de Costa Rica, v. 12, n. 1, 2002.

CHARDIN, Pierre Teilhard de. El fenómeno humano. Paris: Herder, 1970.

CHIAVENATO, Idalberto. Administración en los tiempos modernos. Sao Paulo: McGraw Hill, 2000.

DANIELS, John; RADEBAUGH, Lee. International business: environments and operations. New York: McGraw Hill, 2001.

DUARTE, Klaudio. ¿ Juventud o juventudes?: acerca de cómo mirar y remirar a las juventudes de nuestro continente. Buenos Aires: Editorial Bibios, 2000.

DUBOIS, Alfonso. Un concepto de desarrollo para el siglo XXI. Bilbao, 2005.

ELIZALDE, Antonio. Desarrollo humano y ética de la sostenibilidad. Capítulo 3. Santiago de Chile: PPC, 2003.

FINKIELKRAUT. Citado por Manuel Fernández del Riesgo. En: En torno a la postmodernidad. Barcelona: Anthropos, 1994.

FRANCO, Rosario. La sostenibilidad: una postura autocrítica de la sociedad industrial. Investigación y Desarrollo, Bogotá, v. 13, n. 1, 2005.

FREDERICK, Donald A. Co-ops 101: an introduction to Cooperatives. United States: Madison, 1997.

GILI, Iñaki et al. Memoria de sostenibilidad, aspectos económicos financieros. Bilbao: Deusto, 2005.

GUHL, Ernesto. La dimensión económica del desarrollo sostenible. En: Apuntes para un debate, 2003. Disponible en: <http://www.quinaxi.org>.

HOEXTER, Michael. The concept of sustainability: internal diversity and points of conflict. Futurelab, 2006. Disponible en: <http://blog.futurelab.net/2006/10/the_concept_of_sustainability.htm>.

HOPENHYAN, M. et al. La juventud en Iberoamérica: tendencias y urgencias. 2. ed. Buenos Aires: CEPAL \& OIJ, 2007.

HONORABLE CONGRESO DE LA REPÚBLICA DE COLOMBIA. Ley n. 375 del 4 de julio de 1997. Bogotá.

IICA. Instituto Interamericano para la Agricultura. Concepto de Desarrollo Sostenible. Primera parte, 2007. Revista Electrónica: Infoagro. <http://infoagro.net/shared/docs/a6/PM7.pdf>. 
LÓPEZ, Gustavo. Sostenibilidad planetaria en la era de la sociedad de la información y del conocimiento. Roma: UNESCO, 2003.

LÓPEZ, O. Conceptos y programas para la autogestión. Capítulo 4. Medellín: Panamericana, 1996.

MARGUILIS, Mário; URRESTI, Marcelo. La Construcción Social de la Condición de Juventud. En: Viviendo a toda: jóvenes, territorios y nuevas sensibilidades. Bogotá: DIUC y Siglo del Hombre, 1998.

MORENO, José. Historia y filosofía de la economía solidaria. Una propuesta alternativa, solidaria y humanista. Medellín: Norma, 1995.

OLIVERA, M. El camino de la autogestión ambiental empresarial. Revista Protección y Seguridad. Bogotá, v. 43, n. 249, 1996.

ONU. Informe sobre la juventud mundial. Ginebra: Naciones Unidas, 2005.

Nuestro futuro común. Informe de la Comisión Mundial sobre el Medio Ambiente y el Desarrollo. Ginebra: Naciones Unidas, 1987.

RAZETO, Luis. Los caminos de la economía de solidaridad. Buenos Aires: Lumen-Humanitas, 1996.

ROSENFELD, Stuart. Associative behavior. En: Backing into clusters: Retrofitting public policies. Boston: Harvard University, 2001.

SARMIENTO, Libardo. Memorias del 4to Foro de solidaridad: Asociatividad y Autogestión. Medellín: Confiar, 2005.

SCHNEIDER, José. ¿La educación y la capacitación continúan siendo hoy día opciones importantes en las cooperativas o no? ¿Énfasis mayor en la educación o en la capacitación?. En: Memorias del IV encuentro latinoamericano de investigadores en cooperativismo. Rosario: Universidad de Rosário, 2006.

SCRAMP, Susan. Cooperatives: pathways to economic, democratic and social development in the global economy. Washington: OCDC, 2007.

SENGE, Peter et al. Colaborar para lograr un cambio sistémico. Harvard Deusto Business Review. Bilbao, n. 155, p. 16-28, 2007.

SOUTO, Sandra. Juventud, violencia política y "unidad obrera" en la segunda república española. Madrid: Instituto de Historia del Consejo Superior de Investigaciones Científicas, 2001-2002.

URIBE, Carlos. Bases del cooperativismo. 5. ed. Capítulos 1 y 2. Bogotá: Grupo, 2001.

ZABALA, Salazar Hernando. Las teorías de la solidaridad y el porvenir de la cooperación. CINCOA. Medellín: Latino, 1998.

\section{Referencias de Internet}

ALIANZA COOPERATIVA INTERNACIONAL. <http://www.aciamericas.coop>.

CONFECOOP. <http://www.confecoop.coop>.

DANE. <http://www.dane.gov.co >.

$<$ http://proyecu.galeon.com/cuadernos/cuado/index.html>.

<http://www.aciamericas.coop/spip.php?article45>

CAF. Finanzas sostenibles en América Latina. < http://www.caf.com/publicaciones>.

160 